\title{
El nuevo analfabetismo y la calidad en la
educagión
}

Blanca Ruth Orantes

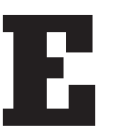
nEl Salvador, existen muchos problemas que superar en materia de educación, aún cuando se han realizado esfuerzos para mejorar el sistema educativo, este sigue lanzando indicadores que visualizan problemas de fondo en materia de calidad, esto lo demuestra el que en la Prueba de Aptitudes y Aprendizajes de Educación Media (PAES) no se han alcanzado calificaciones que reflejan esa calidad ${ }^{1}$ en la mayoría de estudiantes, según fuentes del Ministerio de Educación (Mined). Se puede observar en el comportamiento de rendimiento de los bachilleres en la prueba PAES, que no ha sido significativo el adelanto. ${ }^{2}$ En el 2008 se obtuvo como nota global 6.17 , según datos del Mined.

Lo anterior sin duda repercute directamente en el perfil de entrada con el que ingresan los estudiantes a la educación superior, sea esta pública o privada. Un perfil que en la mayoría no pasa de 7.00 puntos $(70 \%)$ de rendimiento; el restante $30 \%$ no alcanzado refleja, entre otros, problemas de lecto-escritura, dificultades para entender ideas, problemas para redactar y expresarse. A ello se le suma la falta de conocimiento $y / o$ dominio de una segunda lengua; por el momento es el inglés. Se agregan las deficiencias en unos y uso inadecuado en otros en el manejo de paquetes informáticos básicos.

Los análisis que se pueden hacer al respecto son variados, que van desde posiciones radicales que atribuyen la baja calidad de la educación a un modelo excluyente (Freire,1970), hasta los que plantean aumentar la cobertura en educación, sin detenerse en la calidad. Lo que no se puede negar es que la calidad se hace necesaria en todo sistema de educación formal; y es que las exigencias son mayores a escala de país, porque se ha enfatizado que para encontrar un mejor camino al desarrollo, la educación es una ventaja comparativa, pues el conocimiento sirve para insertarse en los procesos de productividad, (Hubbard, 1997).

\section{En nuestro país}

\section{el analfabetismo presenta}

numerosos rostros.

Analfabeto no sólo

es quien no sabe leer

ni escribir; es decir,

incapaz de descifrar

combinaciones de

signos alfabéticos para

transmitir a otros su

sentido.

\footnotetext{
${ }^{1}$ La OCDE (1995) define la educación de calidad como aquella que "asegura a todos los jóvenes la adquisición de los conocimientos, capacidades destrezas y actitudes necesarias para equipararles para la vida adulta".

${ }^{2}$ No significativo, pues no alcanza el promedio por lo menos 7.00 puntos. Obsérvese el comportamiento de las notas globales: 2005: 5.04; 2006: 5.53; 2007: 5.92; 2008: 6.17. Según datos del Ministerio de Educación.

Entorno ISSN: $2218-3345$

Abril 2009, $\mathrm{N}^{\circ} .42$ 
Independientemente de las políticas públicas, la calidad ${ }^{3}$ está vinculada a muchos componentes educativos y culturales; sin embargo, existen dos aspectos que desde mi entender son básicos y necesarios: la formación docente y del alumnado. Antes se mencionó acerca de la formación con deficiencias de los estudiantes de bachillerato, pero también existe el otro problema la calidad docente; la cual también incide directamente en los alumnos. Siendo que el educador estimula al educando para que alcance su propio desarrollo, se está ante un problema trascendental que debe considerarse en cualquier solución o medida posible.

Lacalidaddocentesindudasepuede fortalecer por medio de capacitaciones, no obstante, el problema de fondo es que existen deficiencias en la formación e inserción inicial de muchos profesores relacionadas con el nuevo analfabetismo, que no permiten mejorar significativamente sus competencias docentes y satisfacer las necesidades del proceso enseñanza aprendizaje (Clares, 2000). La calidad de la educación implica un proceso sistemático y continuo de mejora sobre todos y cada uno de sus elementos, principalmente en docentes; existiendo deficiencias de origen, sin duda el reto es mayor, pero lo más grave es que reproducen estas deficiencias en los estudiantes que forman. Estaríamos entonces ante un círculo vicioso que nos aleja de la calidad.

Estas deficiencias tienen mucho que ver con el fenómeno del "nuevo analfabetismo" no en el sentido tradicional de analfabetismo, de no saber leer ni escribir, sino del analfabetismo que implica problemas de lecto-escritura que no es exclusivo de nuestros estudiantes (Canfux, 2003), el analfabetismo que implica la no interpretación adecuada de signos, ignorar o no observar el sistema normativo de convivencia pacífica, entre otros (Lourié,1990).

Una simple descripción comparativa con la realidad observada ubica el nuevo analfabetismo como un problema de grandes repercusiones en la educación y las áreas vinculadas. Por ejemplo, se supone que la educación de los individuos contribuye a una sociedad armoniosa, en la que las personas se respetan a sí mismas, a su entorno, las normas de convivencia pacífica, comprendiendo signos, y comprometidos con el futuro de la sociedad y del país entero; hasta el aspecto ideológico político puede ser superado bajo esta perspectiva, por medio del desarrollo propio en el cual tal como se ha reiterado en

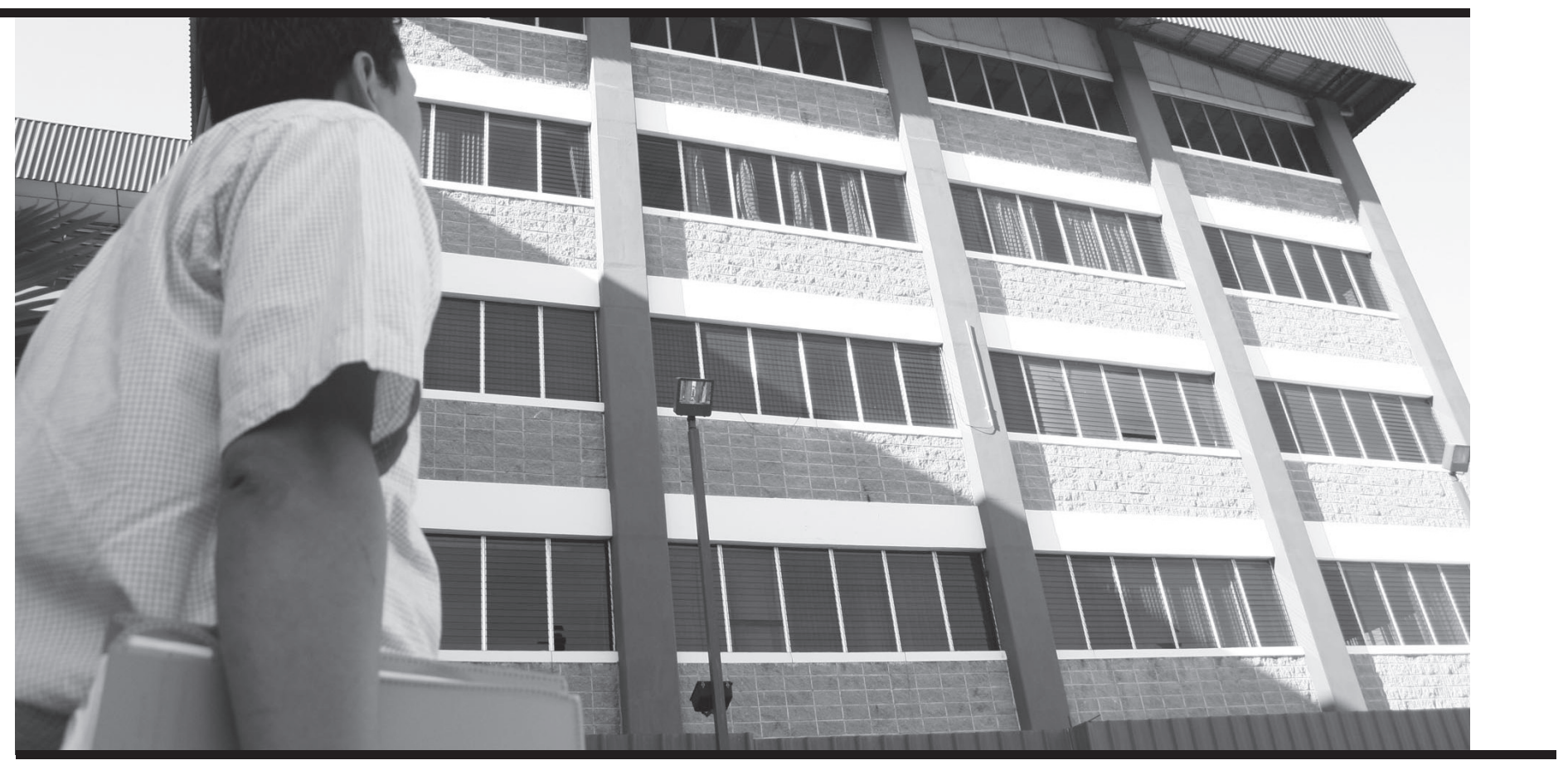

${ }^{3}$ Vinculada en el sentido que "la escuela de calidad es la que promueve el progreso de sus estudiantes en una amplia gama de logros intelectuales, sociales, morales y emocionales, teniendo en cuenta su nivel socioeconómico, su medio familiar y su aprendizaje previo. Un sistema escolar eficaz es el que maximiza la capacidad de las escuelas para alcanzar esos resultados", J. Mortimore (1998) 
muchas ocasiones, la educación es un pilar fundamental que abre las puertas a la productividad y al desarrollo sin depender de otros países (Freire, 1985).

Se dice que el surgimiento de un nuevo analfabetismo sugiere nuevos problemas (Vallejos, 2000). Lo que implica enfocar el problema desde la raíz, situación que conduce a una serie de analfabetismos interrelacionados. Es un fenómeno complejo que se traslada como nuevo analfabetismo al siglo XXI. Dicho fenómeno no es exclusivo de las personas con baja educación, sino también de generaciones que han alcanzado la educación universitaria.

En nuestro país el analfabetismo presenta numerosos rostros. Analfabeto no sólo es quien no sabe leer ni escribir; es decir, incapaz de descifrar combinaciones de signos alfabéticos para transmitir a otros su sentido (Sanzf y Lancho, J., 2001).

Un segundo grado de analfabetismo es el caso de quien puede descifrar los signos alfabéticos, aunarlos y convertirlos en una palabra y ligarla con palabras sucesivas. No obstante, casi todo lo que lee tan solo se compone de anuncios publicitarios en las calles, historietas, la sección
$\ln ^{2}$ la sección
deportiva de los periódicos, literatura religiosa, panfletos y similares. Es lectura obligada, o de esparcimiento, sin disciplina ni el propósito de ampliar horizontes del saber de forma deliberada.

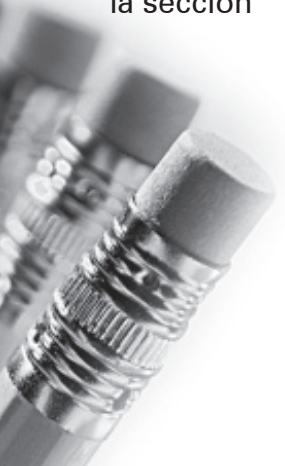

42

\section{Una simple}

descripción comparativa con la realidad

observada ubica el

nuevo analfabetismo

como un problema de

grandes repercusiones

en la educación y las

áreas vinculadas. Por

ejemplo, se supone que

la educación de los

individuos contribuye a

una sociedad armoniosa, en la que las personas se respetan a sí mismas, a

su entorno, las normas

de convivencia pacífica, comprendiendo signos, y comprometidos con el

\section{futuro de la sociedad}

y del país entero.

Dicho analfabeto, en inferior medida, desarrolla la habilidad de expresarse por escrito, de perseguir la profundización y el ordenamiento de su pensar a través de la escritura. Tiene dificultades para entender las ideas y conceptos escritos por otros y para comunicar en forma estructurada los suyos propios. No usa la capacidad de leer y escribir para adquirir y generar conocimientos, sino tan solo pasivamente recibe datos, información aislada y casi siempre trivial y, por lo tanto, carente de utilidad práctica. Los problemas de lecto-escritura que presenta, por ejemplo, la población salvadoreña, incluyendo la que accede a la educación superior universitaria, es un problema de enorme magnitud: puede calificarse como analfabeta funcional.

Un tercer nivel de analfabetismo lo constituye la carencia de conocimiento y habilidades para coordinar acciones con otros en otro idioma, fundamentalmente en inglés. Quien no domina bien este idioma está en serias desventajas y prácticamente no constituye una oferta de servicios (MEC, 1987) tanto para el sector privado como incluso para muchos de los segmentos que ahora conforman el sector público.

El impacto cultural del inglés puede medirse por lo contenido en Internet, que alberga la mayoría de información y conocimientos acumulados por la humanidad; un bajo porcentaje está en otro idioma.

En vista de ello, el español es insuficiente para recibir y transmitir el saber propio de la Revolución del conocimiento, que se sustenta en el saber y la inteligencia. Si se suma la inhabilidad en el terreno informático al desconocimiento del inglés y la ausencia de una óptima comprensión de la propia lengua, lo resul- 


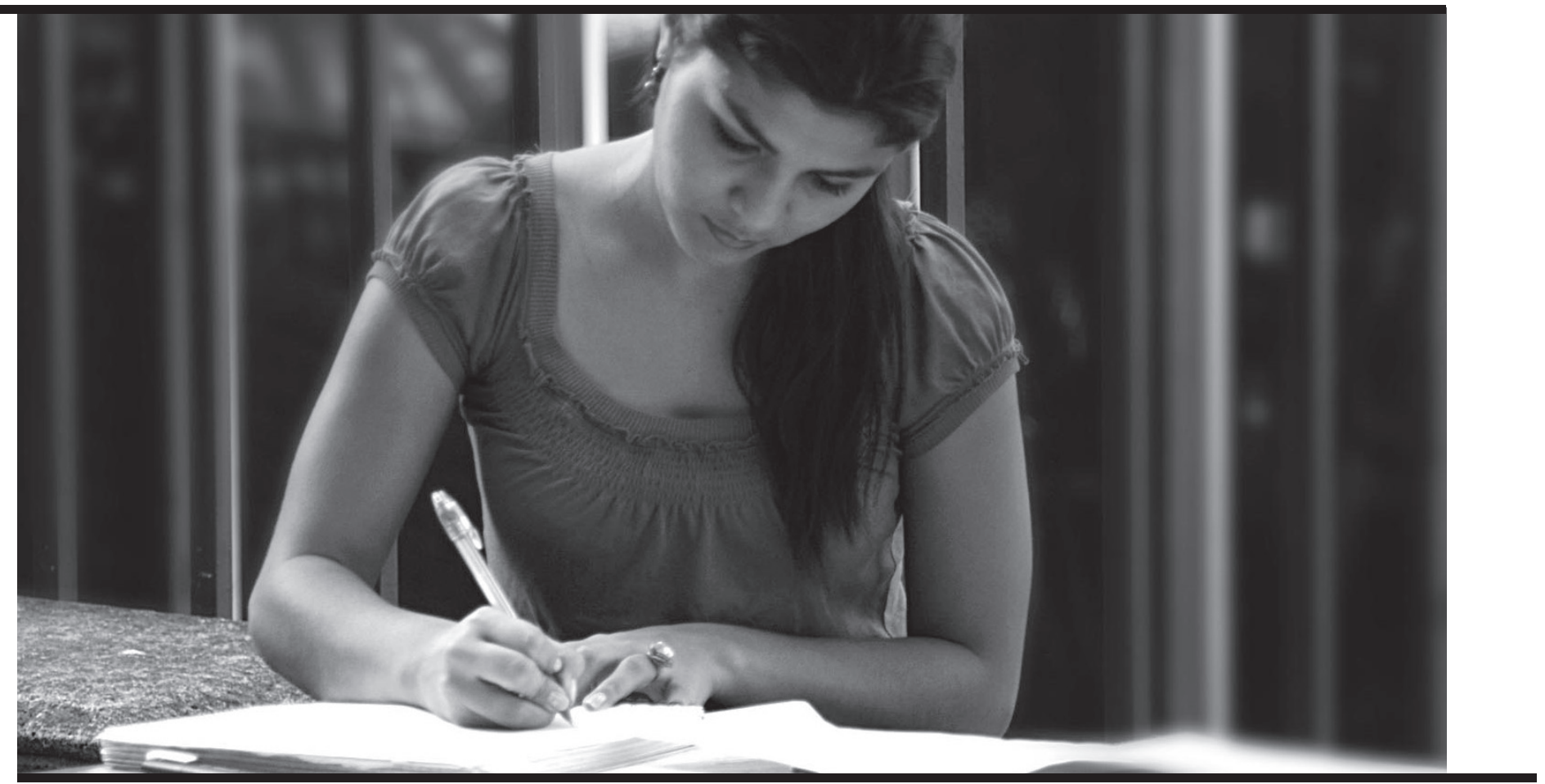

tante sería desde un desempleado potencial hasta un individuo en riesgo de verse convertido en fracasado, parásito social y un peligro para el tejido humano. A este se le denomina analfabetismo multilinguístico.

Hay un cuarto grado de analfabetismo: el informático. Este implica ignorar los significados de términos como Internet, superautopista de la información, multimedias, interactividad, foros de discusión, virtualidad, edición electrónica, digitalización de imágenes, navegación cibernética, buscadores, actividades asistidas o mediatizadas, e incontables otros; y la carencia de las habilidades para manejar paquetes informáticos, abarcando desde procesadores de texto hasta navegadores de la Red, pasando por los editores de imágenes, bases de datos, hojas electrónicas de cálculo, correo electrónico, y muchos otros programas (Aguaded, 2002).
Es de hacer notar que se deben poseer estas habilidades para utilizar instrumentos indispensables para el aprendizaje por cuenta propia y no se diga para el caso de docentes que deben dominar las nuevas tecnologías educativas.

Como puede observarse el nuevo analfabetismo es complejo, porque se está diciendo que existen el analfabeta funcional, el analfabeta multilingüístico y el analfabeta informático. Sin embargo, existen otros géneros de analfabetismo, más cercanos a las actitudes y valores que se deben desarrollar en ellos, todos agrupados bajo el concepto de analfabetismos vinculantes, en el sentido que afectan a la persona humana.

Sumado a lo anterior se tiene el analfabetismo emocional (Freire,
1986), que implica desconocer la existencia de las emociones como predisposiciones fundamentales para la acción y el desempeño humanos, y no comprender la diversidad de reacciones emocionales en cada uno; que los repertorios afectivos que se poseen fueron aprendidos en algún momento de nuestra vida y que, por ende se pueden desaprender $y$, del mismo modo aprender nuevos.

Quienes no entienden y no pueden poner en práctica esto, no pueden diseñar estados emocionales en ellos mismos y en la gente con la que viven y conviven; y desconocen

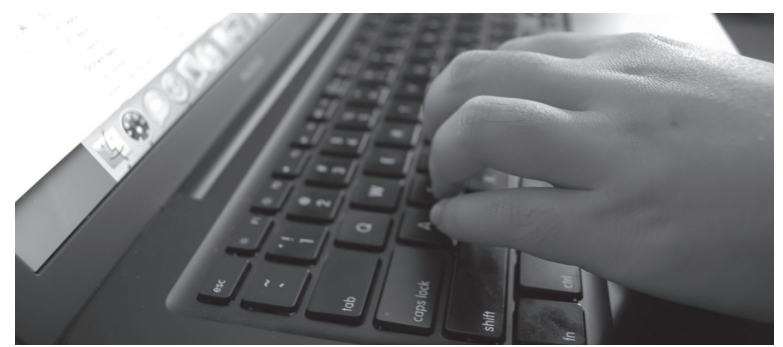




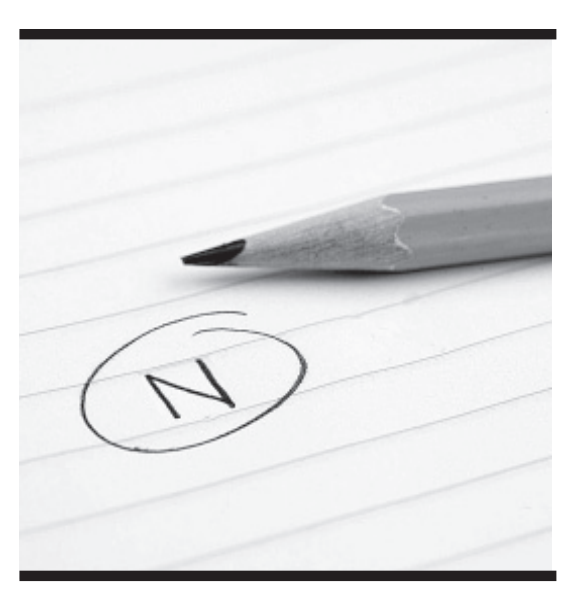

cuáles emociones son las propicias para el trabajo en equipo, el aprendizaje colectivo, y para identificar y solucionar problemas.

También está el analfabetismo conversacional. Cada vez es más valorada la habilidad de una persona para escuchar a otras, de forma interpretativa, captando inquietudes del interlocutor, lo que realmente le interesa, le preocupa y le hace vivir. Por ello el escuchar no es un acto pasivo; al contrario es una habilidad, (Hart, 1986). Por otro lado, está de una forma apropiada; es decir, tener la capacidad de distinguir entre afirmaciones, opiniones y declaraciones: hacer peticiones, ofertas y promesas así como de cumplirlas; de saber emitir juicios sustentados en afirmaciones.

I

El analfabetismo mentecuerpo. Es incapaz de comprender la relación entre sus creencias, emociones y postulados, y el estado de salud que guarda su cuerpo. El cuerpo es la fuente de energía física, mental y espiritual. Trabaja con el cuerpo y para su cuerpo, y no a través de él. Es poco eficaz para aumentar la efectividad de su desempeño laboral a través del trabajo constante sobre el cuerpo en cuanto a su flexibilidad, resistencia, fuerza y relajación. Este tipo de analfabetismo provoca enfermedades que resultan en un alto costo para el sector público y privado, que incluye a los individuos y la familia, afectando su productividad y su vida (Hubbord, 1997).

Por último, se encuentra el analfabeta ecológico. Este es incapaz de compren-der la relación de las partes con el todo, no dejando oportunidades a las generaciones futuras. Implica no poder entender principios de conectividad y organización de los ecosistemas y en una baja capacidad de crear comunidades humanas sustentables y en comprender que su función es el establecer vínculos entre las comunidades ecológicas y humanas, y entre la ecología y la economía. Este tipo de analfabetismo da como resultado el abuso del ecosistema, y afectando así el hábitat de los seres vivos (Vallejos, 2002)

Estas son algunas formas de ver al ser humano en un contexto evolutivo. Los procesos de enseñanzaaprendizaje que no tomen en cuenta e incluyan estos aspectos en su práctica educativa cotidiana, tienden a ser poco efectivos e inútiles. Poblaciones como la salvadoreña, no podrían salir del abismo del subdesarrollo y del atraso cultural en que está sumida en medio de la pobreza, el hambre y la desesperanza si no logran saltar las grandes barreras del analfabetismo que bajo sus diversas formas obstaculizan su avance.

Hay muchas víctimas del analfabetismo bajo cualquiera de sus manifestaciones. Las universidades reciben docentes y jóvenes y adultos con esta variedad de analfabetismos, que no es solo el tecnológico, porque de nada sirve querer enseñar inglés y computación a los universitarios, si no se está consciente de las otras formas incidentes de analfabetismo de las que son víctimas, y que en

\section{Como puede}

observarse el nuevo

analfabetismo es

complejo, porque

se está diciendo que

está el analfabeta

funcional, el analfabeta

multilinguiístico y el

analfabeta informático.

Sin embargo existen otros

géneros de analfabetismo,

más cercanos a las

actitudes y valores que

se deben desarrollar en

ellos, todos agrupados

bajo el concepto de

analfabetismos

vinculantes, en el sentido

que afectan a la persona

humana.

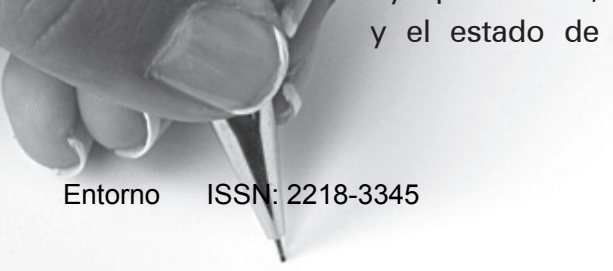


alguna medida no permiten alcanzar la calidad académica, que no solo depende de la universidad, sino de la educación parvularia, básica y media.

\section{Cada procedimiento de aprendizaje} necesita un tipo de herramientas, primero se tiene que tener claro que la globalización del conocimiento nos enfrenta a una frontera tecnológica incierta, de cambios irreversibles en la biología humana y en la ecología del mundo. Se requiere de un tipo de educación en tecnología radicalmente diferente, que permita tomar decisiones inteligentes en ese tipo de futuro (González, 2007).

Los avances técnicos suelen traer aparejado más de un efecto, ya que abren un nuevo universo de posibilidades; y ese mismo universo traza un límite restrictivo que permite el ingreso de unos a la vez que excluye a otros. La revolución informática no ha sido una excepción. Está ha cambiado el horizonte de nuestra cultura, proporcionando herramientas inimaginables en el pasado, que van desde la conquista española hasta el alto nivel de transculturación y alienación en la sociedad salvadoreña ya en el siglo XXI.

Pero también con dicha revolución y los fenómenos subyacentes se ha creado un mundo de marginados de esos recursos. $Y$ es que para la mayoría de los hogares pobres y de baja educación en El Salvador el acceso a la computadora y a internet es muy lejano, y la exclusión se hace cada vez más latente; pero aparejado a este vienen otros tipos de analfabetismo, que hacen aún mayor el abismo entre países desarrollados y subdesarrollados, marcando aún más la dependencia en todo sentido.

Las universidades reciben jóvenes y adultos con esta variedad de analfabetismos, tanto en el sector do-cente como en el estudiantado. Debido a ello, de nada sirve querer enseñar inglés y computación, si son víctimas de analfabetismos vinculantes que, en alguna medida, no permiten alcanzar la calidad académica, que no solo depende de la universidad, sino también de la educación inicial (parvularia básica, mediay de formación profesional).

Esta inquietud dio origen, en el 2008, a iniciar un estudio sobre "El nuevo analfabetismo y su impacto en los estudiantes universitarios", como parte de la labor investigativa en la Universidad Tecnológica de El Salvador, el cual se concluirá en el año 2009, esperando conocer datos cuantitativos y cualitativos de este fenómeno en las universidades del país. Partiendo de los hallazgos, se hará una propuesta que busque aplicar medidas para minimizar este fenómeno $y$, paralelamente saltar este obstáculo en el camino a la calidad académica y como efecto de cascada, a solucionar otros problemas vinculantes del entorno social, laboral y del medio ambiente.

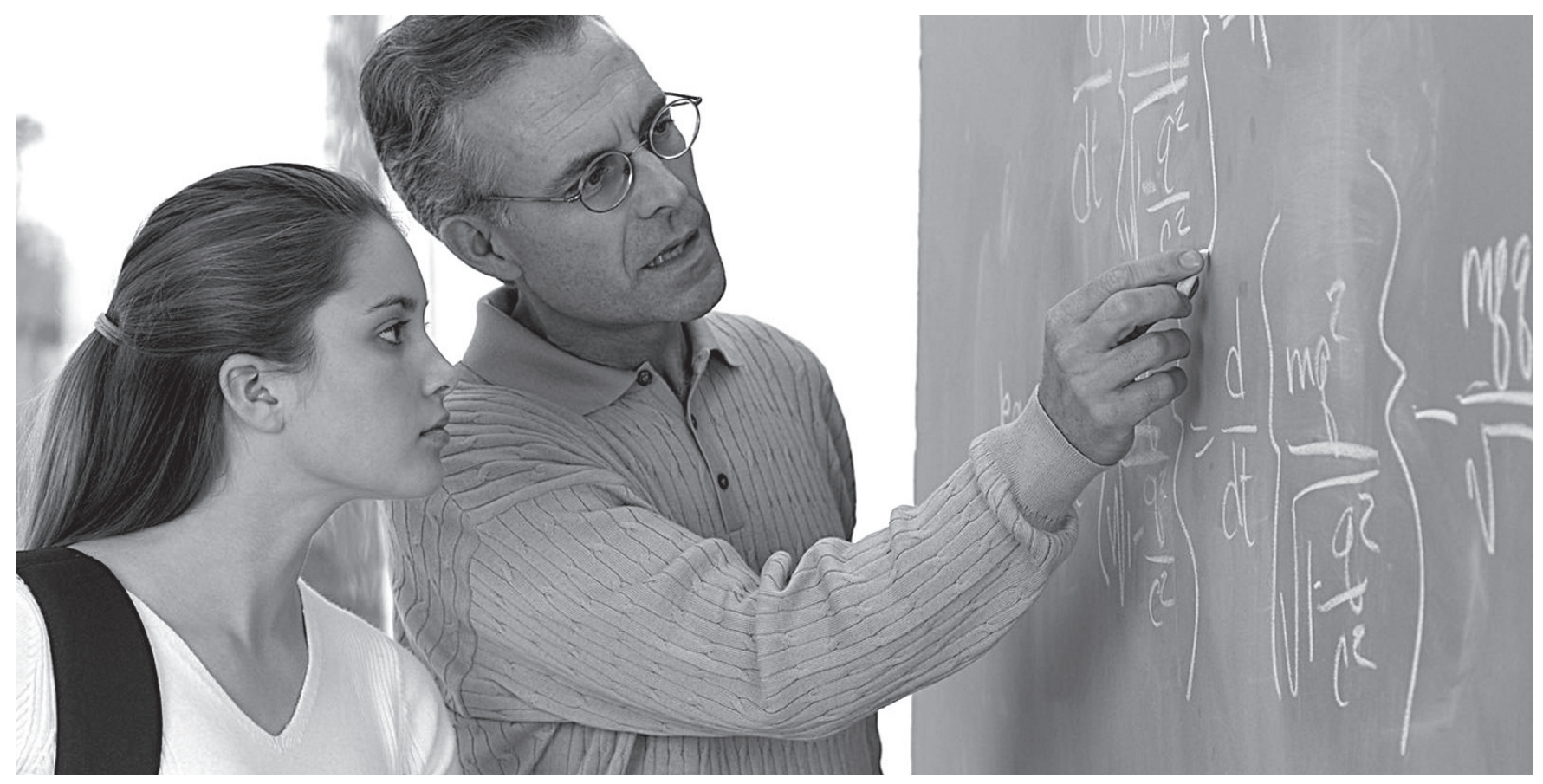




\section{Conclusiones}

Sin haber concluido la investigación, ya se percibe que una gran parte de la población salvadoreña en general, y la universitaria en particular, está afectada por el nuevo analfabetismo, lo que significa un llamado urgente para aunar esfuerzos y minimizar este fenómeno.

El nivel de afectación puede asociarse con la crisis social, en la que se incluyen problemas graves como la salud en donde, si bien es cierto hay que mejorar las políticas públicas, también hay responsabilidad de la población, que no aplica hábitos sanos de alimentación y otras actividades afines que están vinculadas al analfabetismo mentecuerpo. Pueden citarse también los graves problemas del medio ambiente, como el de la basura y la contaminación, que refleja un analfabetismo ecológico. Los dos fenómenos citados no son ajenos al comportamiento de la población universitaria salvadoreña, que debería marcar la diferencia.

En resumen, se presume que existe un alto porcentaje de analfabetismo funcional en la población universitaria, por el comportamiento observado.
Si no se pone "el ojo de águila" en este problema no se podrán erradicar muchos problemas en la sociedad salvadoreña $y$, particularmente, en el sector universitario, que atenta contra la calidad académica en todo sentido.

\section{Reflexiones finales}

El Salvador es parte del contexto globalizado, que se caracteriza por un incesante devenir de cambios económicos, culturales y sociales; donde el empleo de las nuevas tecnologías de la información y la comunicación son indispensables para alcanzar el éxito profesional; donde las actitudes y los valores son imprescindibles para lograr el desarrollo personal, y donde los sistemas de relaciones son inherentes a la integración social de los individuos. Lo anterior significa que hay que reconocer las formas del nuevo analfabetismo para mejorar paulatinamente los niveles de calidad y cumplir, por medio de la educación, primero en el sistema educativo y luego a escala nacional.

Lasuniversidades debenbuscarlaraíz del problema y hacer lo suyo, sin dejar de investigar otras causas en educación básica y media, proporcionando un aporte en investigación educativa con recomendaciones y propuestas viables para todo el sistema en todos los niveles de educación formal. Lo anterior podría abrirnos nuevas perspectivas a nivel regional e internacional al mejorar los niveles de calidad.

En este contexto, existe un compromiso compartido entre el Estado y las organizaciones, la sociedad civil, los sectores educativos, en donde las universidades son entes importantes en este país, que deben corregir a fondo este fenómeno que se reproduce como células cancerosas y que atenta negativamente contra nuestro futuro como país. Ese compromiso conlleva apostarle a la educación integral básica conforme a las necesidades en cada nivel de escolaridad y sector destinatario del producto educativo que se forma (laboral, urbano y rural). No obstante, esa educación integral debe desarrollarse en un sistema educativo de calidad, incluyente, pero que a la vez forme a las nuevas generaciones (docentes $y$ estudiantes) con capacidades para poder competir en este mundo globalizado y, a la vez, transformen el comportamiento de subdesarrollo que ha caracterizado a países como el nuestro.

\section{BIBLIOGRAFÍA}

- AGUADED, J.l. y CABERO, J. Educar en Red. Málaga, Aljibe. 2002

- CANFUX, J. MARBOT, E. "Metodología para la educación". Curso precongreso Pedagogía 2003. La Habana, Cuba

- CLARES, JOSÉ. Orientación educativa y NNTT. Comunicación y Pedagogía. CALIDAD ENEDUCACION http://dewey.uab.es/PMARQUEs/calida2htm. 04/12/2008.

- FREIRE, P. La importancia de leer y el proceso de liberación. Editorial Siglo XXI, Cuarta Edición. Ciudad de México. 1986.

- FREIRE, P. La educación como práctica de la libertad. Editorial Siglo XXI. 32 . Edición. Ciudad de México. 1985.

- GONZALEZSANMAMED, Mercedes. "Las TIC comofactor de innovaciónymejora delacalidad delaenseñanza". En CABERO, Julio. TecnologíaEducativa. Madrid: McGrawHill. 2007.
- HART, C. Analfabetismo científico en la nuera era imperial. Artículosy documentos ajenos. Asociación Cultura Pazy Solidaridad Haydee Santamaría. La Habana: Noviembre, 2003.

- HUBBARD. LR. La llave de la vida. Disponible en: Chururh Of Scientology Intemacional. 1999.

- LOURIE, S. El verbo y la acción. Revista UNESCO. 1990.

- MEC. "Proyecto para la reforma de la enseñanza. Propuesta para debate". Madrid. Ministerio de Educación y Ciencia. Madrid, España. 1987.

- OCDE Schooling for tomorrow: trenes and scenarios. Paris: CERI-OECD. 2001.

- SAMOMONS, HUMAN, MORTIMORE. "Características clave de las escuelas efectivas". Secretaría de Educación Pública. México. 1998.

- SANZFyLANCHO,J. "Informe basesobrelaeducaciónyformación de personas adultas en España". Examentemático delaeducación de adultos dela OCDE. 2001.

- VAUEJOS, AMPARO. "Luces en el laberinto audiovisual". Congreso lberoamericano de Comunicación y Educación. 2005. 
